\title{
Computational Modeling of Liquid and Gaseous Control Valves
}

\author{
R. Daines* \\ Jacobs Sverdrup NASA Test Operations Group, NASA Stennis Space Center, MS 39529 \\ and \\ V. Ahuja, ${ }^{\dagger}$ A. Hosangadi, ${ }^{\star}$ and J. Shipman ${ }^{\S}$ \\ Combustion Research and Flow Technology, Inc. (CRAFT Tech), Pipersville, PA 18947 \\ and \\ A. Moore $^{* *}$ and P. Sulyma ${ }^{\dagger \dagger}$ \\ Louisiana Tec, and NASA Stennis Space Center, MS
}

\begin{abstract}
In this paper computational modeling efforts undertaken at NASA Stennis Space Center in support of rocket engine component testing are discussed. Such analyses include structurally complex cryogenic liquid valves and gas valves operating at high pressures and flow rates. Basic modeling and initial successes are documented, and other issues that make valve modeling at SSC somewhat unique are also addressed. These include transient behavior, valve stall, and the determination of flow patterns in LOX valves. Hexahedral structured grids are used for valves that can be simplifies through the use of axisymmetric approximation. Hybrid unstructured methodology is used for structurally complex valves that have disparate length scales and complex flow paths that include strong swirl, local recirculation zones/secondary flow effects. Hexahedral (structured), unstructured, and hybrid meshes are compared for accuracy and computational efficiency. Accuracy is determined using verification and validation techniques.
\end{abstract}

\section{Introduction}

The ability to accurately model flow through a control valve is important to the support of rocket engine component testing that occurs at NASA Stennis Space Center. Along with cavitating venturis and orifices, control valves provide the ability to test engineers to control flow rates and pressures in the test system. One especially critical role that control valves play is in the startup and shutdown timing of propellant delivery rates and pressures to the test article. Getting the proper mix of fuel and oxidizer during these transient periods of operation is critical for the safety and operation of rocket engine components. Because of a lack of understanding of the exact response of propellant delivery systems and control valves in particular, numerous time-consuming and expensive activation runs must be conducted to characterize the test system. If key components of that system, such as valves, can be accurately modeled, it has the potential to decrease the time and cost to test, while at the same time increasing safety and confidence.

The work detailed in this paper is an overview of the work carried out over the last three years at SSC in the area of control valve modeling. Axisymmetric models will be discussed first, along with their advantages and disadvantages, as well as guidelines as to when they are suitable. 3-D models will be discussed. These have been

\footnotetext{
* Senior Research Scientist, Jacobs Sverdrup NASA Test Operations Group, Stennis Space Center, Senior AIAA Member.

${ }^{\dagger}$ Research Scientist, 6210 Keller's Church Road, Pipersville, PA 18947, Senior AIAA Member.

* Principal Research Scientist, 6210 Keller's Church Road, Pipersville, PA 18947, Senior AIAA Member.

${ }^{\S}$ Research Scientist, 6210 Keller's Church Road, Pipersville, PA 18947, AIAA Member.

** Graduate Student, Louisiana Tech, Ruston, LA, Student Member.

${ }^{\dagger}$ Senior Research Scientist, NASA, Stennis Space Center, MS, Senior AIAA Member
} 
extremely useful in a better understanding of the detailed flow physics that occur in control valves. Both cryogenic liquid valves, modeled either as an incompressible flow, or with real fluid effects and compressible gas valves will be discussed. Some future directions will also be discussed including transient the valve modeling, tailoring valve trim sets above the test requirements, and potential future are as to expand our valve analyses.

\section{Methods}

Two CFD codes available at SSC were used and tested on valve flows in the present work. One is FDNS, a pressure-based code developed for NASA Marshall Space Flight Center ${ }^{1,2}$ which employs a structured flow solver. It has been used for modeling propulsion related flowfields at MSFC for many years. Parallel execution can be accomplished using either PVM or MPI. Real-fluid properties are modeled in FDNS by using a lookup table based on curve fits of a set of discrete data points from a comprehensive real-fluids model to retrieve thermodynamic properties.

The second code available at SSC is CRUNCH CFD ${ }^{\circledR}$ code, which has a hybrid, multi-element unstructured framework that allows for a combination of tetrahedral, prismatic, and hexahedral cells ${ }^{3}$. Grids for complex 3D control valves are generated very efficiently by dissolving the seat region and inlet/discharge ducts by hexahedral and prismatic cells, and stitching them together with tetrahedral cells ${ }^{4,5}$. The grid connectivity is stored as an edgebased, cell-vertex data structure, where a dual volume is obtained for each vertex by defining surfaces that cut across edges coming to a node. An edge-based framework is attractive in dealing with multi-elements since dual surface areas for each edge can include contributions from different element types making the inviscid flux calculation "grid transparent".

For efficient computation of large 3D problems, a parallel framework for distributed memory systems has been implemented, along with a time-marching implicit solution procedure. The sparse implicit matrix is derived using an Euler explicit linearization of the first-order flux, and a variety of iterative sparse matrix solvers, e.g. GMRES, Gauss-Seidel procedure, are available in the code ${ }^{6,7}$. The parallel framework is implemented by partitioning the grid into sub-domains, with each sub-domain residing on an independent processor. The message passing between processors has been implemented using MPI to provide portability across platforms. The CRUNCH CFD ${ }^{\mathbb{B}}$ code has an integrated grid refinement and coarsening package operational within a multi-element framework ${ }^{8}$. In addition to traditional regimes of incompressible and highly compressible flows, CRUNCH CFD ${ }^{\circledR}$ code also has embedded models for real fluid effects and cavitation ${ }^{9}$.

\section{Results}

\section{A. 2-D Axisymmetric Modeling of Cryogenic Liquid Control Valves}

The geometry of control valves can be very complicated and in every case varies in all three dimensions. The first step in modeling these valves at SSC was to simplify the 3-D valve geometry to a somewhat-equivalent axisymmetric model, provided that the important flow physical phenomena is unaffected by the approximation. For the plug and seat region, an axisymmetric model is geometrically correct, while for the rest of the valve it is an approximation. The geometry upstream and downstream of the seat was modified to make it axisymmetric for the model. The area modeled started slightly upstream of the valve seat and extended about six duct diameters downstream, which is sufficient to allow the flow to realign with the duct and pressure to recover.

Figure 1 shows the pressure contours over a range of plug positions in an axisymmetric cryogenic control valve model. The seat-region geometry of this valve had been modified at SSC for LOX service, and the effect of these changes on the valve performance needed to be quantified before testing. At plug positions $55 \%$ open or less, a region of large pressure drop extends along the length of the seat and extends over the nose of the plug, with the greatest pressure drop occurring at the trailing corner of the upstream face of the seat. This corner corresponds to the minimum flow area for these configurations. The character of the flow begins to change at $55 \%$ open. The trailing edge of the plug, which is more tapered, is opposite part of the seat at this plug location. The low pressure region no longer extends over the entire length of the seat as it does for smaller valve openings.

Figure 2 shows the streamlines at three plug positions. The region of the valve near the valve seat and plug are shown, including the space in the center of the plug. The bottom of each figure is the centerline of the nozzle. The Coanda effect keeps flow attached to the nose of the plug and directs it toward the centerline of the valve. This creates a recirculation zone near the valve body immediately downstream of the seat over the entire range of plug positions. The separation point occurs at the downstream face of the valve seat. 
AIAA-2005-4543
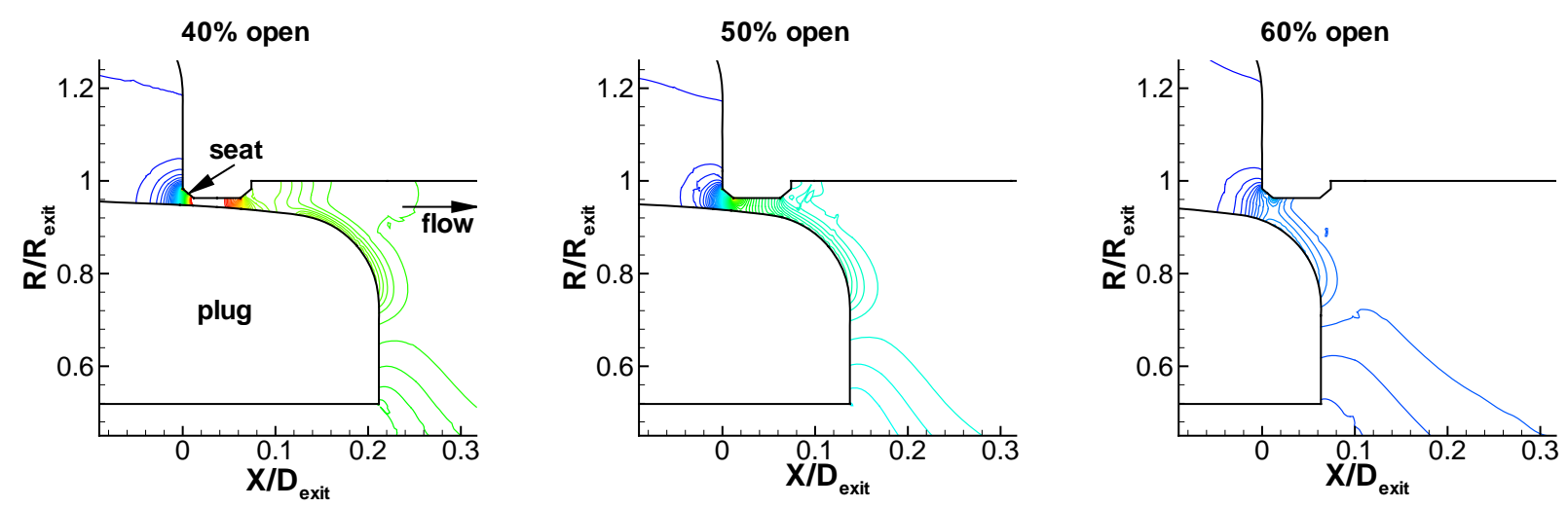

Figure 1. Normalized Pressure Contours in an Axisymmetric Value Model Using FDNS.
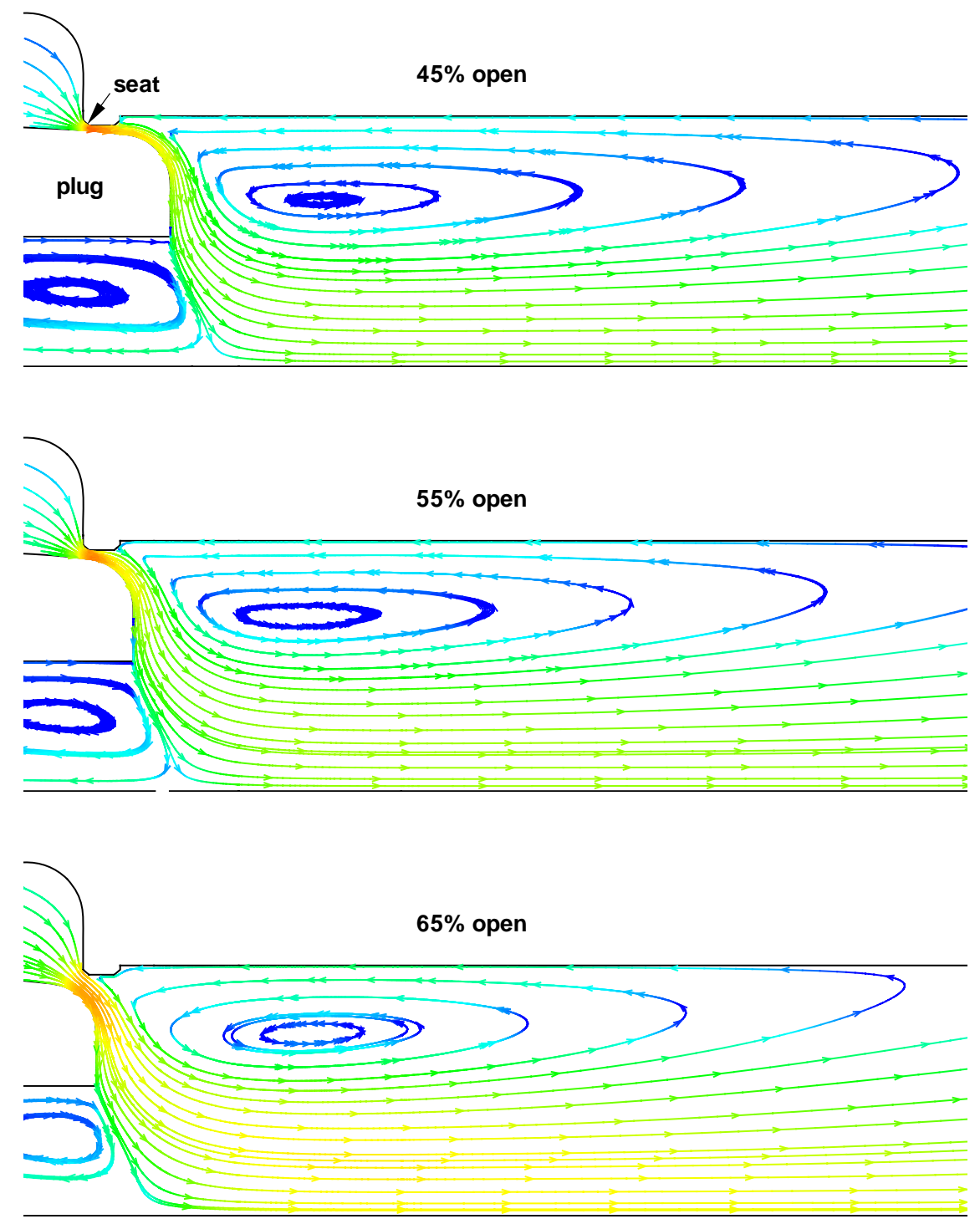

Figure 2. Streamlines for a Range of Plug Positions in an Axisymmetric Valve Model Using FDNS. Color indicates Velocity Magnitude, with Red Indicating Higher Velocities and Blue Indicating Lower Velocities. 
Figure 3 shows the global results of this modeling effort in terms of the valve coefficient, $C_{v}$ as a function of valve position. To calculate $C_{v}$ the integrated mass flow rate at the inlet and the difference of averaged pressures between the inlet and full pressure recovery point downstream of the plug were used

$$
C_{v}=\frac{\dot{m}}{\sqrt{\Delta P}}
$$

It is noted that this is a dimensional number. The results for the two codes are very close. Although an exact comparison is not possible since the experimental data was taken at slightly different valve openings, the interpolated numerical results are typically close to the experimental measured values between 40 and $70 \%$.

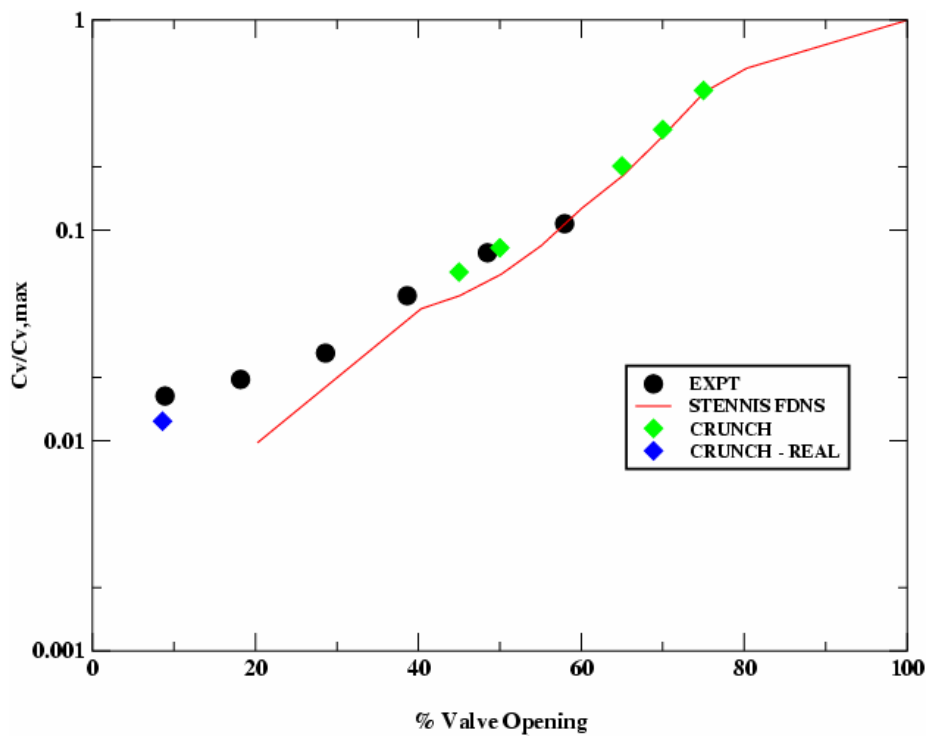

Figure 3. Valve Coefficient $C_{v}$ as a Function of Plug Position. Calculated and Experimental Test Values are Shown.

The axisymmetric single fluid model breaks down for this valve both at the higher openings $(>70 \%)$ and at the plug positions where the valve is near completely closed $<20 \%$ ). At the larger opening, pressure losses die to 3 -D effects such flow turning and swirl generated from flow around the plug dominate pressure losses in the seat region. At the lower openings real fluid effects take over and density changes to the liquid as a result of pressure gradients in the seat region how to be accurately accounted for. Figure 3 shows this effect at the $8 \%$ open position where real fluid effects were accounted for in the CRUNCH CFD ${ }^{\circledR}$ calculation. It should be noted that the y-axis is plotted in log scale here showing extremely close agreement between the experimental data and the CRUNCH calculation. The FDNS results with an incompressible single fluid approximation start showing a deviation from experimental data from a plug position of $40 \%$ and mass flow errors dominate at setting lower than $20 \%$.

For code verification, three grids were generated at the $65 \%$ open position with approximately $15,500,60,000$, and 232,000 grid points, that is, the grids were doubled in each direction ${ }^{10}$. The error bands were calculated using Richardson Extrapolation. The results using single- and double-precision arithmetic is shown in Figure 4. With double-precision arithmetic, the error bars for the 60,000 point grid are about $\pm 4 \%$, while the single precision error is around $\pm 9 \%$. 


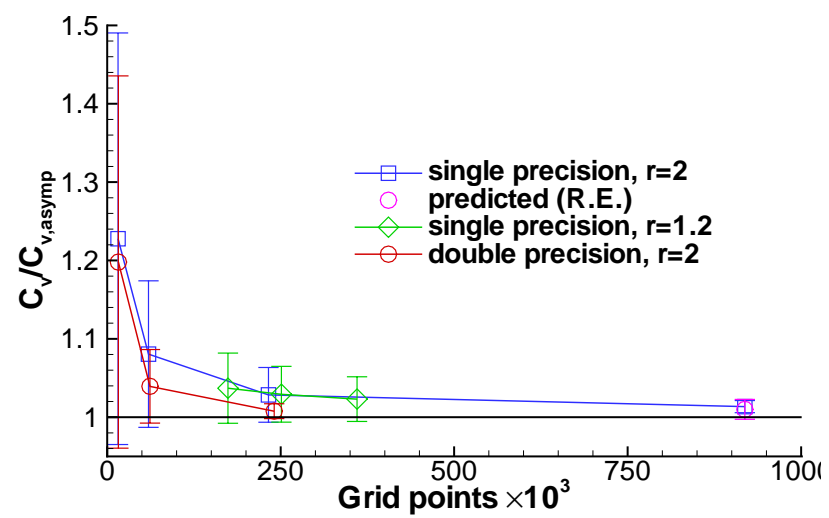

\section{Figure 4. Normalized Valve Coefficient for Different Grid Densities, Showing the Calculated Numerical Error Bands for Each Point Compared to the Theoretical Grid Independent Value of $C_{v}$ from Richardson Extrapolation.}

When working with complex geometries such as control valves, it is advantageous to be able to use unstructured or multi-element grids to reduce the time and complexity of the gridding procedure. To study the effects of the grid type on the solution, a structured, an unstructured, and a multi-element grid were generated for one valve position. Unstructured grids here refer to grids consisting only of tetrahedral and prismatic elements, while multi-element grids also include hexahedral elements. The analysis results can be seen in Figure 5. Because the boundary layer is important in predicting the flow losses through the valve and especially in the seat region, the structured grid yields the highest quality solution. It can be seen that the results from the unstructured grid are significantly different than those of the structured grid. The high-speed stream downstream of the plug has a different shape, and the recirculation zones downstream of the seat are much more compact. This is in spite of the fact that the unstructured grid has significantly more nodes than the structured grid: 390,000 tetrahedral and 122,000 prismatic cells compared to 60,000 hexahedral cells. The predicted valve coefficient is about $4.5 \%$ low compared to the structured grid, as shown in Table 1. In addition, the unstructured grid required 8 times as much CPU time as either of the other approaches to produce a solution. With a multi-element grid, hexagonal cells are placed in the boundary layers where boundary layer resolution is critical, with tetrahedral and prismatic elements upstream and downstream of the seat region. These grids are almost as easy to generate as an unstructured grid, but it can be seen that the results are much better. Multi-element results are nearly indistinguishable from the results from the structured grid, and the valve coefficients in Table 1 are nearly the same. These results indicate that we are able to use multi-element grids in complex 3-D situation and maintain high-quality analysis solutions.

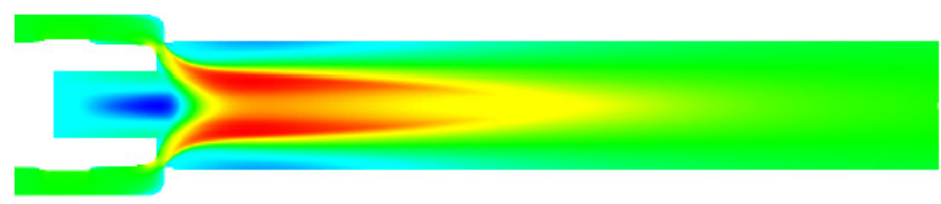

(a). Structured

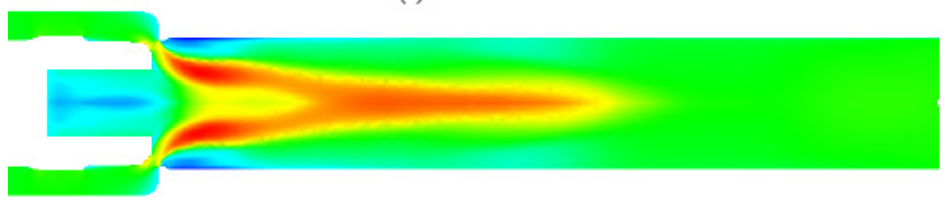

(b). Unstructured

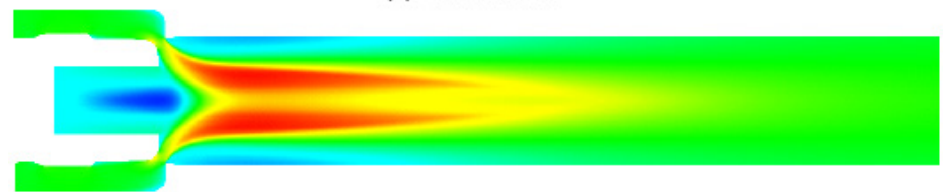

(c). Multi-Element

VELOCITYTX]

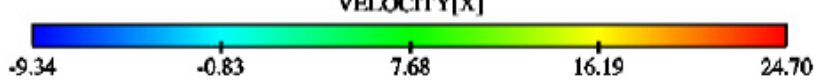




\section{Figure 5. Velocity Contours in an Axisymmetric Liquid Valve on Three Types of Grids: Structured,} Unstructured, and Multi-Element.

Table 1. Normalized Valve Coefficients for Three Grid Types at 75\% Open

\begin{tabular}{cc} 
Grid type & $\frac{C_{v}}{C_{v, \max }}$ \\
\hline Structured & 0.462 \\
Multi-element & 0.463 \\
Unstructured & 0.441
\end{tabular}

Local grid refinement was attempted to improve the unstructured flow solution. The grid adaption technique used in the CRUNCH CFD ${ }^{\circledR}$ framework is a Delaunay based refinement procedure ${ }^{11}$. Grid adaption improved the solution significantly. Flow patterns closely resemble those captured in the hexahedral and multi-element solutions. However, the adapted grid has almost three times the number of tetrahedral cells as the original grid, further increasing the required CPU time for a solution.

An additional area of work is focused on being able to model the behavior of control valves under dynamic conditions. This is important in a test stand environment where transient operation of the test facilities can take a significant fraction of the total test time. The work covering this aspect of valve modeling including a description of the grid motion methodology is reported in a companion paper ${ }^{12}$.

The valve modeled above has a plug profile typical of a valve type referred to as an "equal percentage plug", which indicates that the percentage change in mass flow with valve position is constant through much of the operating range of the plug. This gives a $C_{v}$ curve characterized by the curve shown in Figure 3. Another general type of plug profile used is a "linear plug". In a valve with this type of plug, the $C_{v}$ curve is approximately linear with percent open. To do this the plug tapers much more rapidly, allowing the flow area in the seat region to open up more quickly.

Another valve referred to as the split-body valve was built and tested for use at Stennis which had a linear plug. It was modeled to help gain greater understanding of valve modeling and where our models were successful. The valve design featured exchangeable trim sets (plug and seat combinations) so that different flow ranges could be achieved using a single valve body (Figure 6). Axisymmetric modeled for both a full-bore and reduced-bore trim sets of the valve were developed and run over a range of valve openings from 5 to $100 \%$. The $C_{v}$ curve for the fullbore trim set with flow over the plug is shown in Figure 7. (Flow over the plug indicates the flow enters the seat region after flowing around the plug, while flow under the plug indicates the flow goes through the seat region and encounters the nose of the plug first before it flows around the plug.) It can be seen that the numerical models begin to break down at about 20 to $30 \%$ open, and by $100 \%$ open the numerical model over predicts $C_{v}$ by more than a factor or two. The breakdown occurs even earlier in the reduced-bore model. The lack of ability of the axisymmetric model to adequately capture the flow physics at these much smaller valve openings occurs because of the plug shape. This is the point where flow losses in the seat no longer dominate the total flow losses in the valve and 3-D effects become important. A general modeling rule that can be drawn from the modeling of these two valves is that axisymmetric models are applicable over a much wider valve opening range for equal percentage geometries than for linear geometries.

One other problem was noted while modeling this valve; FDNS was unable to conserve mass at small valve openings. At valve openings less than around $30-40 \%$ mass conservation errors exceeded $5 \%$, with the error increasing as the valve opening decreased. Because of this, the FDNS results in this regime cannot be considered quantitative. Several efforts to correct the problem were unsuccessful, including running the valve with laminar flow. CRUNCH CFD ${ }^{\circledR}$ did not exhibit any mass conservation problems. 

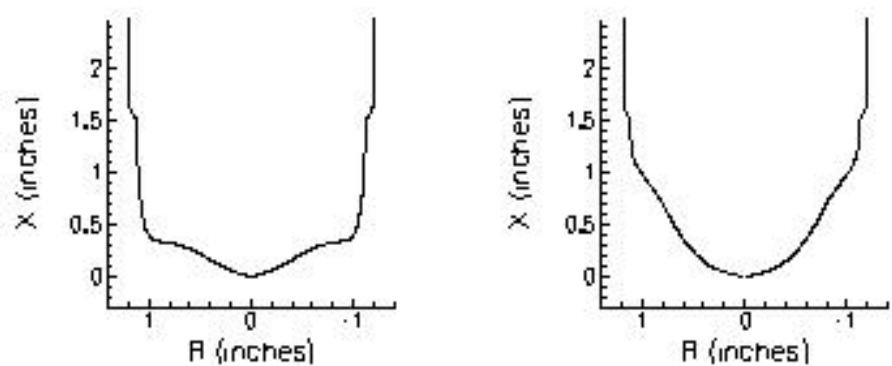

Peduced.Bore, Orıgınal Plug

Reduced-Bore, Modilied Plug
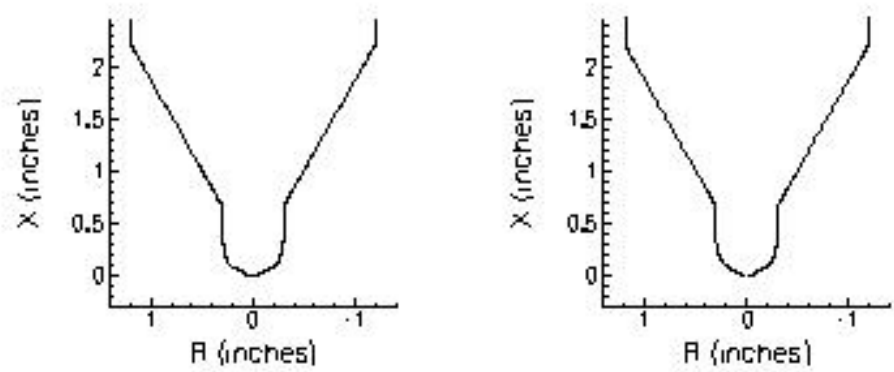

Figure 6. Valve Designs With Different Trim Sets Showing the Full Bore and the Reduced Bore Plugs.

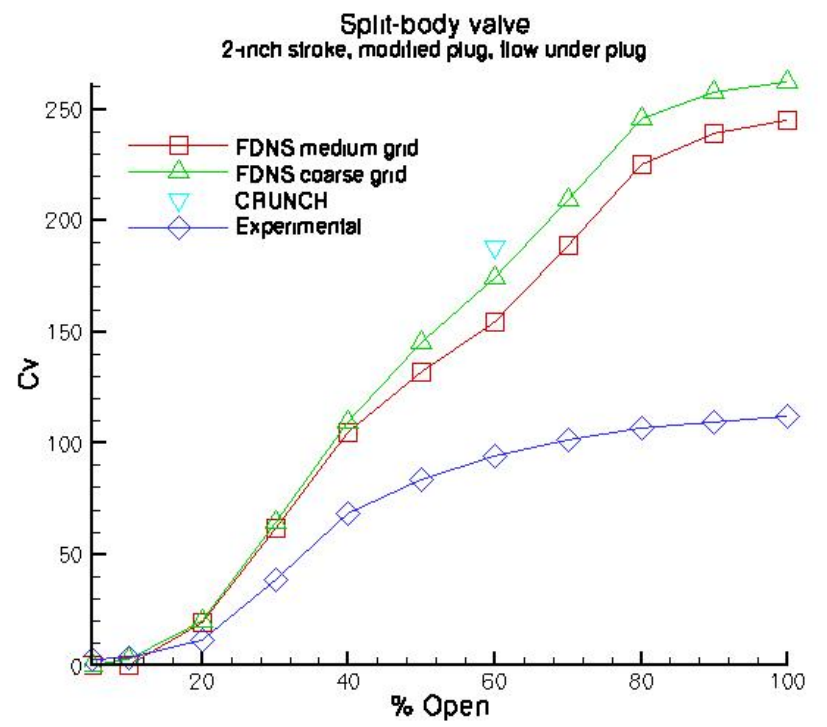

Figure 7. $C_{v}$ Curve For Full Bore Trim Set With Axisymmetric Approximation.

\section{B. 3-D Modeling of Cryogenic Liquid Control Valves}

Since using an axisymmetric approximation to predict $C_{v}$ on these types of control valves break down when the losses in the seat region no longer dominate the valve losses, 3-D modeling is required to more accurately predict valve losses at larger valve openings. In addition, 3-D modeling is the only way to get details of flow patterns in the valves. A 3-D model of full-bore split body valve was generated at $100 \%$ open. To reduce the grid count, a symmetry plane is included along the vertical centerplane of the valve. Figure 8 shows the streamlines for this 
control valve for flow under the plug. It can be seen that the flow patterns vary significantly from those typical in $2-$ $\mathrm{D}$ axisymmetric models. In particular, the primary path of the flow is below the plug in this case, with a recirculation zone forming in the region on the upper side of the plug. This in part explains why the 2-D approximation breaks down; flow patterns cease to be axisymmetric in the seat region. The other reason is that the losses which the valve flow coefficient measures, along with not exhibiting axisymmetry, are no longer concentrated in the seat region but are spread throughout the valve. An accurate representation of the geometry upstream and downstream of the seat becomes important. It can be seen from the streamlines that significant flow losses result from the 3-D effects, such as the flow turning the corner on the lower part of the seat and the recirculation zone in the upper part of the plug cavity. It was noted above that the axisymmetric model overpredicted $C_{v}$ by more than a factor or two. Table 2 shows that the full 3-D model still over predicts $C_{v}$ by $10 \%$ for FDNS and about $20 \%$ with CRUNCH CFD ${ }^{\circledR}$. Work is being pursued to increase the accuracy of the models.

Table 2. Experimental and Numerical Valve Coefficients for Fl9ow Under the Full-Bore Split-Body Valve at $\mathbf{1 0 0 \%}$ Open

\begin{tabular}{ccc} 
& $C_{v}$ & Deviation \\
\hline Experimental & 112 & - \\
FDNS & 124 & $10 \%$ \\
CRUNCH & 134 & $20 \%$
\end{tabular}
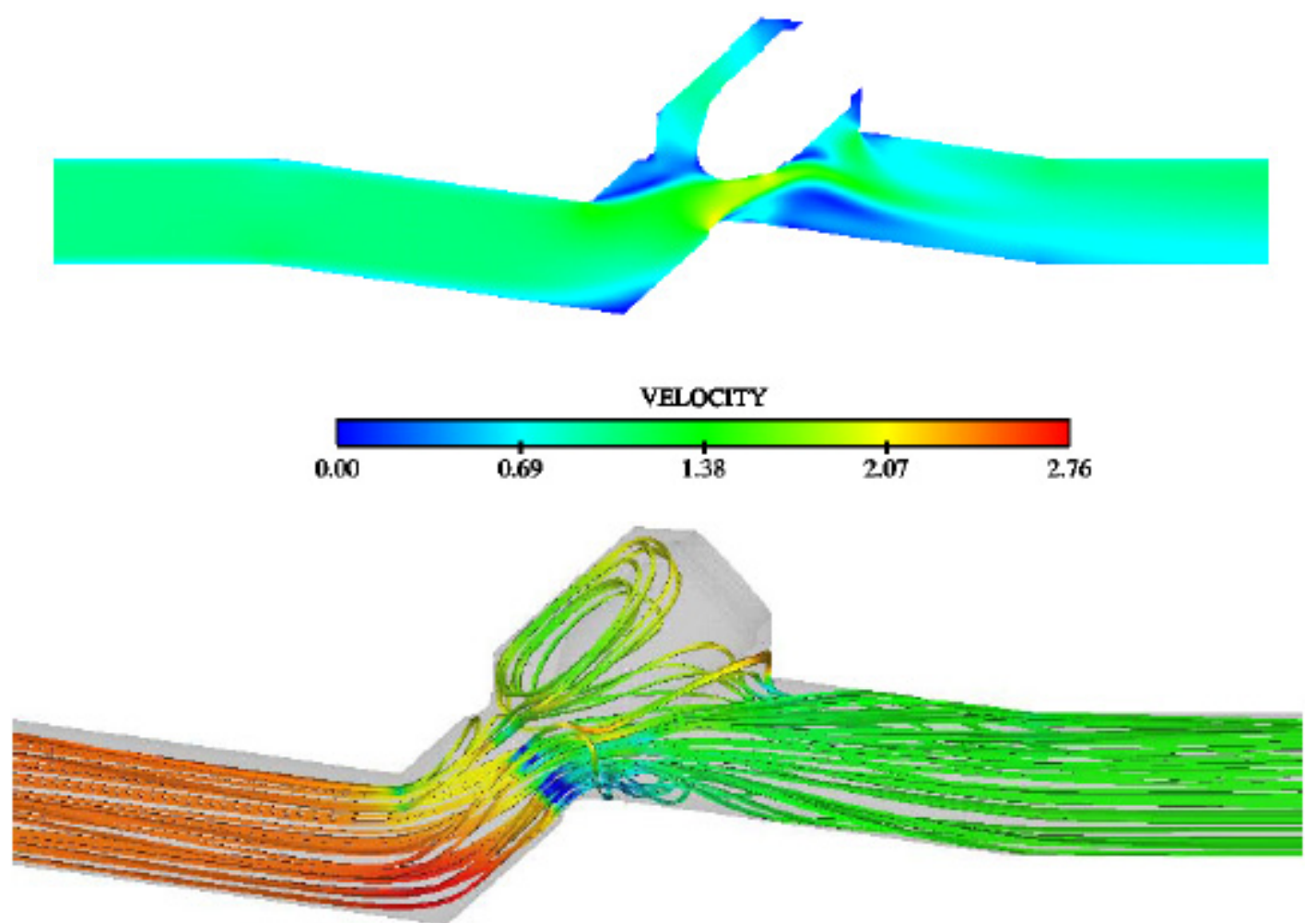

Figure 8. Velocity Contours and Streamlines Colored by Pressure in a Liquid Valve.

\section{Modeling Of A High-Pressure Gas Valve}

Work has also been done to model flow of high-pressure gases through y-pattern control valves. Work has previously been reported on modeling a high-pressure gas regulator valve ${ }^{5}$. Because of the high-speed flows which can occur in high-pressure gas valves, compressibility effects become important. Compressibility and other gas effects cause flow patterns to vary significantly from the cryogenic liquid valve counterparts. With the pressure ratios used across valves in systems at SSC when throttling the flow, the flow tends to choke in the seat region. One test system configuration included a 6-inch gaseous control valve that would stall at about $52 \%$ open during the shutdown process. A flow analysis was done to help determine the cause of the stalling. An axisymmetric model of 
the control valve (not shown) indicates supersonic flow is achieved slightly downstream of the plug in a valve operating at $6000 \mathrm{psi}$. It also indicated that, unlike the liquid valves, the flow would tend to stay towards the walls of the downstream duct rather than flowing towards the center of the duct. However, the results were insufficient to determine the cause of the stalling.

To get higher fidelity, a steady 3D model of this valve was developed. As with the liquid 3-D valve model, the grid uses the vertical half-plane symmetry to reduce the number of grid points. Results from this model are shown in Figure 9. As in the liquid control valve, the highly 3-D nature of the flow can be seen in the figure. There is a significant amount of swirling downstream of the plug. It also can be seen that supersonic flow and a series of Mach diamonds occur downstream of the plug and seat. This model was used to calculate the aerodynamic forces on the plug during operation.

The pressure on the plug surface, shown in Figure 10 was integrated

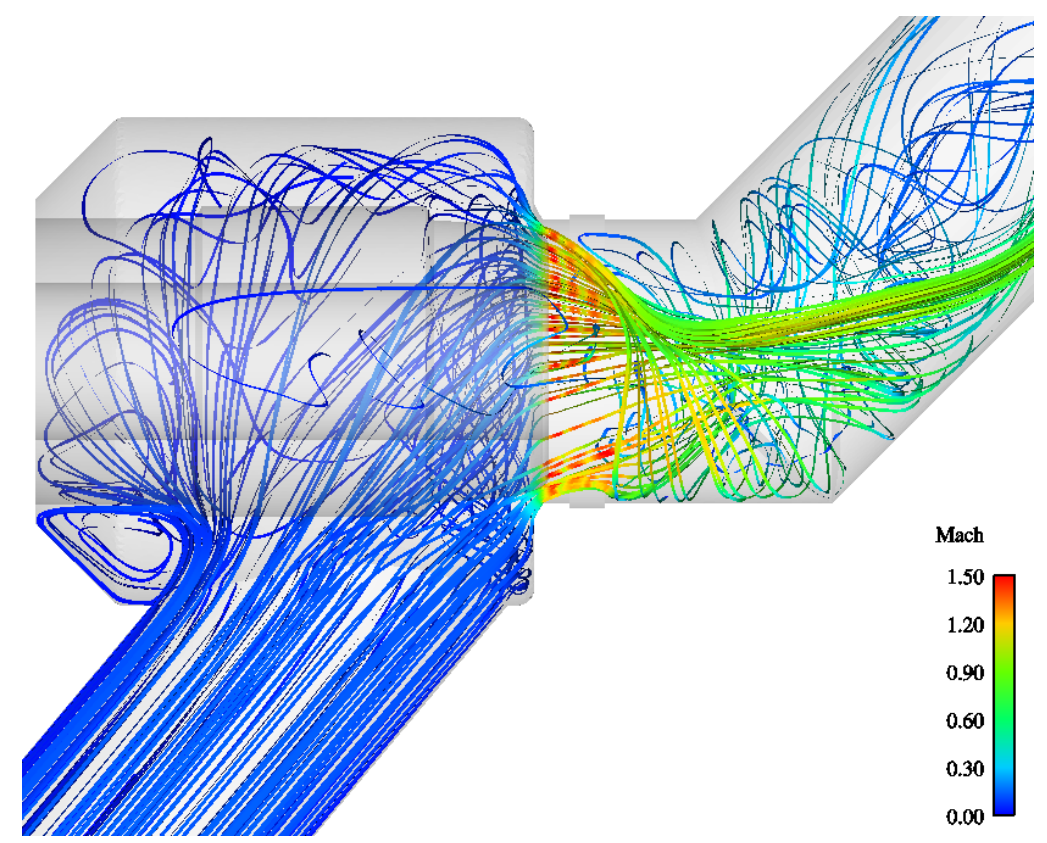

Figure 9. Streamlines Colored by Mach Number in a High Pressure Gas Valve. to determine if the valve stall problem that had occurred was likely due to the forces from the low pressure region on the downstream side of the plug. Because the flow is supersonic, the pressure upstream of the minimum area point in the plug-seat region is significantly higher than the pressure in the region of the expanded flow. The valve studied here is a balanced plug, which means the pressure at the center of the face of the plug is transmitted upstream to a balance chamber. When the flow shocks, the pressure at the outer edge of the plug upstream of the choke point is much higher than the pressure in the balance cavity. This causes a net upward pressure force on the plug. If the annular ring of high pressure forces on the plug extended far enough toward the center of the plug, the upward aerodynamic force exerted on the plug could overwhelm the hydraulic actuator at high enough velocities. The force that could be exerted by the hydraulic system on the plug to close it was $12,000 \mathrm{lbf}$. This analysis yielded an upward force of 9,000 lbf, which was insufficient to be the cause of the stalling.

Unsteady simulations were performed utilizing the MILES (Monotone Integrated Large Eddy Simulation) methodology with the multi-element unstructured CRUNCH CFD ${ }^{\circledR}$ code to further investigate the stall problem. The simulations were performed with identical conditions as the steady simulations discussed above. The time step utilized in these simulations was set at $2 \times 10^{-7}$ seconds. A sequence of instantaneous plots depicting Mach number and pressure distributions is shown in Figures 10-11. These plots are 500 iterations or $10^{-4}$ seconds apart. The general flow features such as the flow expansion in the seat region are quite similar to those seen in the steady simulations. However, the key difference in the unsteady simulations is the temporal flapping of the jet past the plug. As a consequence the dynamics of the compression/expansion wave structure in the seat region is altered. The sequence of instantaneous Mach number distribution and pressure plots (Figures 11) on the symmetry plane clearly depict the flapping of the jet and periodic vortex shedding in the discharge duct. The jets flow through the seat region and coalesce downstream in the valve housing and pressure waves travel through the plug cavity. The interaction of the traveling pressure waves in the cavity with the flow in the valve housing causes pressure transients in the valve housing that affect the dynamics of the under-expanded jets flowing through the valve seat causing the jets to break in symmetry and flap. In other words, the plug cavity does not represent a benign flow region as is generally the case in such valve configurations, but rather behaves like a resonant tube with pressure waves from the cavity interacting with the flow in the seat region. This in turn breaks the symmetry of the flow in the seat region and leads to variable loads on the plug. 

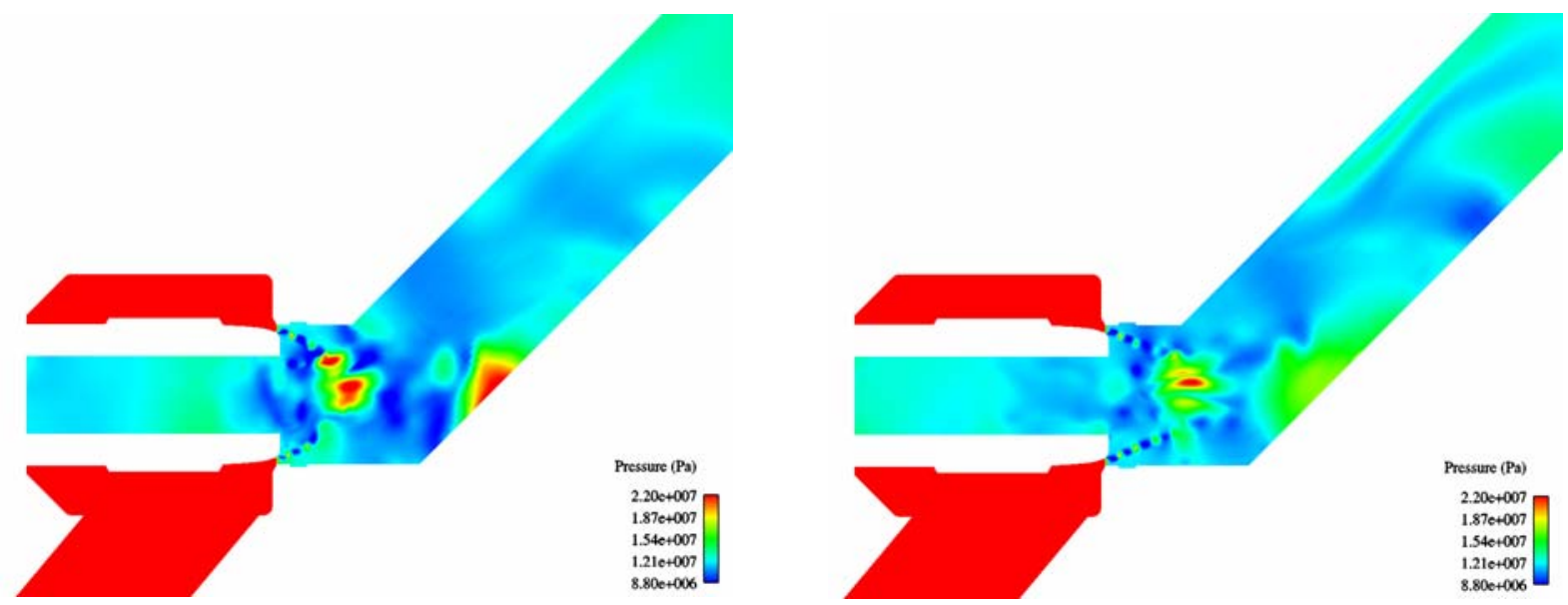

Figure 10. Instantaneous Pressure Distribution in 6-inch gaseous Hydrogen Valve.
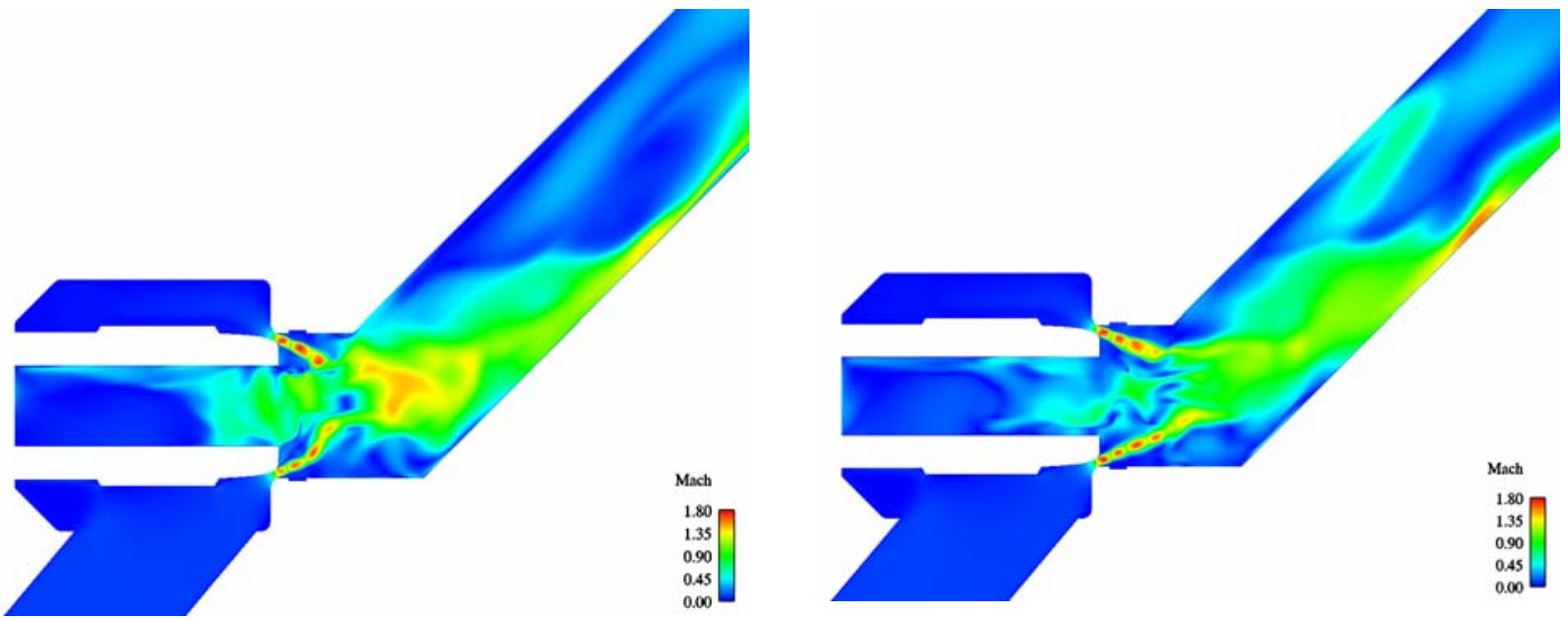

Figure 11. Instantaneous Mach Number Distribution in 6-inch gaseous Hydrogen Valve.

The time history of the axial force on the plug is plotted in Figure 12. Each count on the $\mathrm{x}$-axis is 10 time iterations or $2 \times 10^{-6}$ seconds and portions above the $12,000 \mathrm{lbf}$ on the $\mathrm{y}$-axis are colored indicating that the force on the valve plug has exceeded the force rating on the actuator leading to valve stall. The figure shows dramatic fluctuation in the force of more than $3000 \mathrm{lbf}$ and explains the valve stall seen during testing. Two assumptions need to be stressed at this point: first, the frequency excited in the plug cavity is dependent on the length of the cavity. The length of the cavity considered in the computational domain is different from that used in testing. It was shortened for computational purposes to make the problem computationally tractable, and a priori the effect of the cavity on valve stall was unknown. Second, in actual testing there will be feedback from valve stall on the flow physics of the jet and the flow in the cavity. That feedback

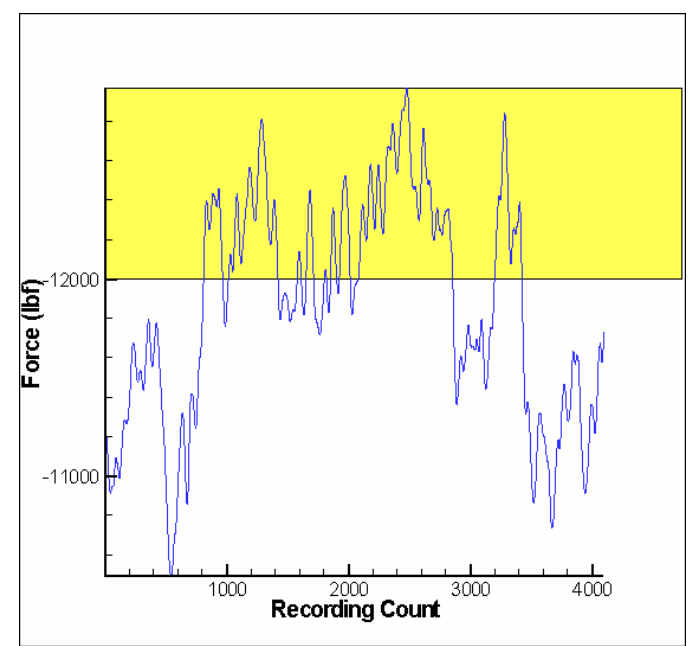

Figure 12. History of the axial force on the plug. (1 unit on the $x$-axis is 10 time iterations or $2 \times 10^{-6}$ seconds). 
cannot be accounted for in a pure CFD simulation at a constant plug setting. These assumptions, notwithstanding the unsteady simulations helped elucidate the physics behind valve stall and the instability mechanisms responsible for it.

\section{Summary and Future Directions}

Y-pattern control valves have been modeled at Stennis Space Center with both cryogenic liquid and highpressure gas as the working fluid. Because of the modeling that has been accomplished, 3-D effects are better understood in both the liquid and gaseous flow regimes. Techniques have been developed to more quickly generate grids and achieve solutions to these problems. Guidelines have been developed regarding when simple axisymmetric models are sufficient to yield the desired information. Because the flows through valves can be affected by dynamic pressure fluctuations, time-accurate modeling of the valves can yield results that are more physically realistic.

\section{Acknowledgments}

We would like to recognize and thank the many people who have supported the valve modeling work at SSC in a number of ways. Some of these include, in no particular order, Shamim Rahman, David Coote, Robert Field, Jared Sass, William St Cyr, Jody Woods, Gregory McVay, Lester Langford, William Mark Mitchell, Nickey Raines, Haynes Haselmeyer, Michael Yentzen, Kern Witcher, Robert Bruce, John Bailey, and John Lansaw.

\section{References}

1 Chen, Y., "Compressible and Incompressible Flow computations With a Pressure Based Method," AIAA Paper No. AIAA89-0286, $27^{\text {th }}$ Aerospace Sciences Meeting, Reno, NV, Jan. 9-12, 1989.

2 Daines, R.L., Woods, J.L., and Sulyma, P.R., "Progress in Valve Modeling at Stennis Space Center," Penn State Propulsion Engineering Research Center Fourteenth Annual Symposium, State College, Pennsylvania, December 2002.

3 Ahuja, V., Shipman, J.D., Arunajatesan, S., and Hosangadi, A., "Multi-Element Unstructured Methodology for Analysis of Turbomachinery Systems, Journal of Propulsion and Power, Vol. 19, No. 5, pp. 945-952, Sept-Oct 2003.

4 Ahuja, V., Hosangadi, A., Shipman, J., Daines, R., and Woods, J., "Multi-Element Unstructured Analyses of Complex Valve Systems, Accepted for Publication, Journal of Fluids Engineering, 2005.

5 Ahuja, V., Hosangadi, A. and Shipman, J., "Multi-Element Unstructured Analyses of Complex Valve Systems," 52 ${ }^{\text {nd }}$ JPM/1 ${ }^{\text {st }}$ LPS Meeting, Las Vegas, NV, May 10-14, 2004.

6 Hosangadi, A., Lee, R.A., York, B.J., Sinha, N., and Dash, S.M., "Upwind Unstructured Scheme for Three-Dimensional Combusting Flows," Journal of Propulsion and Power, Vol. 12, No. 3, pp. 494-503, May-June 1996.

7 Hosangadi, A., Lee, R.A., Cavallo, P.A., Sinha, N., and York, B.J., "Hybrid, Viscous, Unstructured Mesh Solver for Propulsive Applications," AIAA-98-3153, AIAA 34th JPC, Cleveland, OH, July 13-15, 1998.

8 Hosangadi, A. and Ahuja, V., "A Generalized Multi-Phase Framework For Modeling Unsteady Cavitation Dynamics And Thermal Effects," Paper No. AIAA-2003-4000, 33 ${ }^{\text {rd }}$ AIAA Fluid Dynamics Conference, Orlando, FL, Jun 23-26, 2003.

9 Ahuja, V., Hosangadi, A., Ungewitter, R. and Dash, S.M., "A Hybrid Unstructured Mesh Solver for Multi-Fluid Mixtures," AIAA Paper No. 99-3330, 14 ${ }^{\text {th }}$ AIAA CFD Conference, Norfolk, VA, June 28-July 1, 1999.

10 Daines, R.L., Woods, J.L., and Sulyma, P.R., "Computation Analysis of Cryogenic Flow Through a Control Valve," FEDSM-2003-45120, 4th ASME_JSME Joint Fluids Engineering Conference, Honolulu, Hawaii, July 2003.

11 Cavallo, P.A., and Baker, T., "Efficient Delaunay-Based Solution Adaptation for Three-Dimensional Unstructured Meshes," AIAA Paper No. 2000-0809, $38^{\text {th }}$ AIAA Aerospace Sciences Meeting at Reno, NV, Jan 10-13, 2000.

12 Ahuja, V., Hosangadi, A., Cavallo, P.A. and Daines, R. "Analyses Of Transient Events In Complex Valve and Feed Systems," Paper No. AIAA-2005-4549, $41^{\text {st }}$ AIAA/ASME/SAE/ASEE Joint Propulsion Conference \& Exhibit, 10 - 13 Jul 2005, Tucson, Arizona. 